\title{
Transformation between polar and rectangular coordinates of stiffness and dampness parameters in hydrodynamic journal bearings
}

\author{
Zhuxin TIAN $^{1}$, Yu HUANG ${ }^{1,2, *}$ \\ ${ }^{1}$ School of Mechanical Engineering, Hubei University of Arts and Science, Xiangyang 441053, China \\ ${ }^{2}$ State Key Lab of Digital Manufacturing Equipment and Technology, School of Mechanical Science and Engineering, Huazhong University \\ of Science and Technology, Wuhan 430074, China \\ Received: 05 May 2019 / Revised: 26 August 2019 / Accepted: 11 September 2019 \\ (C) The author(s) 2019.
}

\begin{abstract}
The stiffness and dampness parameters of journal bearings are required in rectangular coordinates for analyzing the stability boundary and threshold speed of oil film bearings. On solving the Reynolds equation, the oil film force is always obtained in polar coordinates; thus, the stiffness and dampness parameters can be easily obtained in polar coordinates. Therefore, the transformation between the polar and rectangular coordinates of journal bearing stiffness and dampness parameters is discussed in this study.
\end{abstract}

Keywords: coordinate transformation; stiffness parameters; dampness parameters; hydrodynamic journal bearings

\section{Introduction}

In modern industry, rotating parts of engineering equipment are supported by journal bearings in the vertical direction. The machine characteristics are significantly dependent on the performances of journal bearings. Due to the oil whip effect in a rotating hydrodynamic journal bearing, self-excited vibration occurs in the oil film, which increases with an increase in the rotation speed [1-4]. As a result of the self-excited vibration, threshold speed and stability boundary exist for the rotating bearings. When the rotating speed is larger than the threshold speed, the vibration results in large orbiting amplitudes of the journal and leads to the contact between the journal and bearing, causing bearing failure. Further, the bearing is stable at the journal bearing center in the stability boundary. Khonsari and Chang [5] analyzed the nonlinear stability of journal bearings, and obtained the stability boundary by tracking the journal center trajectory. To easily derive the linear threshold speed, Huang et al. [6] chose polar coordinates instead of Cartesian coordinates to signify the state vector. Lin et al. [7, 8] discussed the threshold speed and stability boundary of hydrodynamic journal bearings lubricated using non-Newtonian fluids. Kushare and Sharma [9] dealt with the threshold speed of two lobe symmetric hole entry worn hybrid journal bearing by theoretically considering the non-Newtonian behavior of the lubricants.

The threshold speed and stability boundary of the hydrodynamic journal bearing are determined based on the stiffness and damping coefficients. Lund and Thomsen [10] proposed a method to study these coefficients. The Reynolds equation is derived with respect to Cartesian coordinates and stiffness and damping coefficients are obtained by integrating the new equations. Based on this method, Jang and Kim [11] studied the dynamic characteristics of journal bearings with five degrees of freedom (DOF) and derived the perturbation equations. Crooijmans et al. [12] discussed the self-excited vibration of hydrodynamic journal bearings, and interpreted the dynamic performance

* Corresponding author: Yu HUANG, E-mail: yhuanghust@163.com 


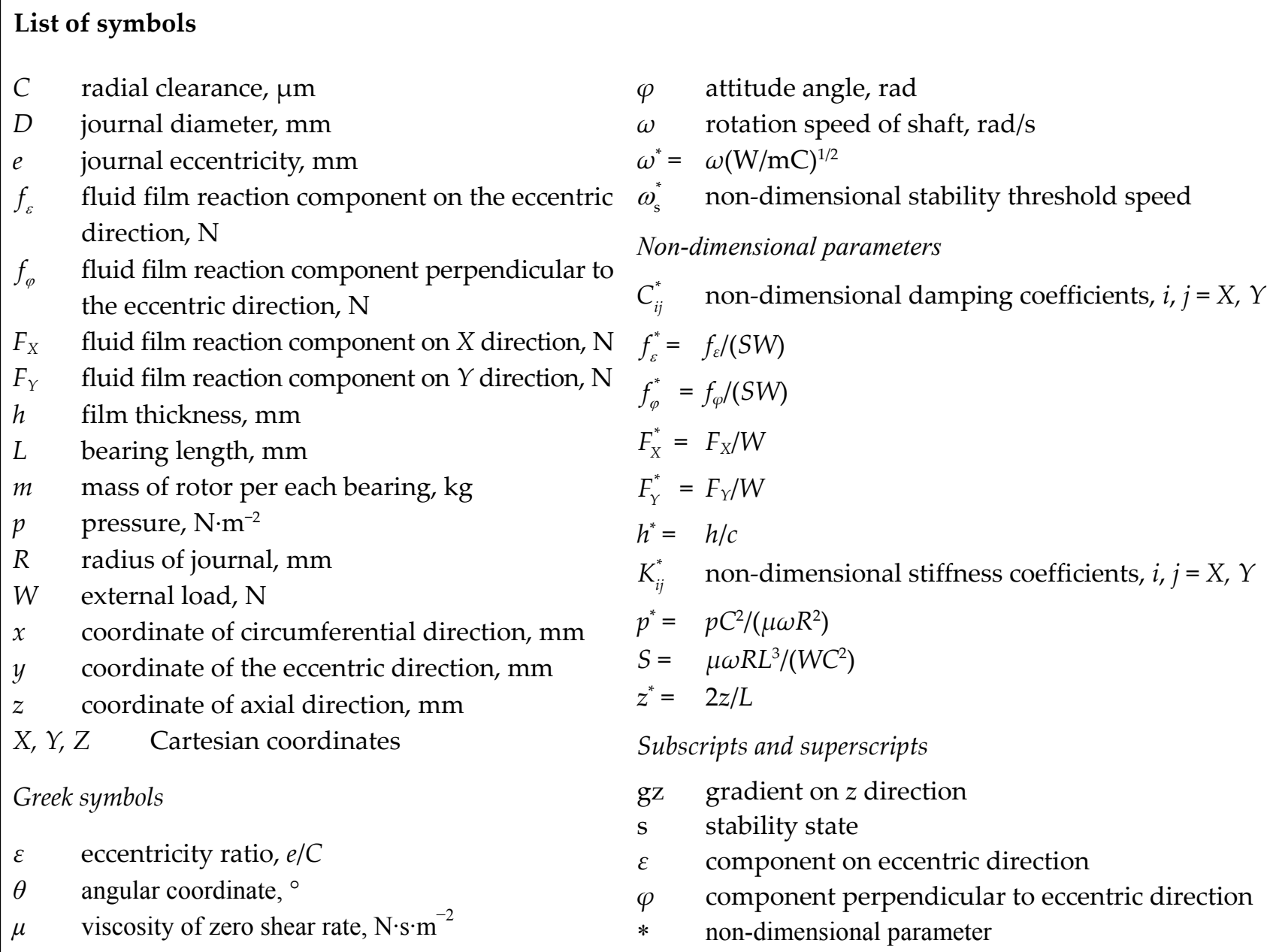

$\varphi \quad$ attitude angle, rad

$\omega$ rotation speed of shaft, $\mathrm{rad} / \mathrm{s}$

$\omega^{*}=\omega(\mathrm{W} / \mathrm{mC})^{1 / 2}$

$\omega_{s}^{*} \quad$ non-dimensional stability threshold speed

Non-dimensional parameters

$C_{i j}^{*} \quad$ non-dimensional damping coefficients, $i, j=X, Y$

$f_{\varepsilon}^{*}=f_{\varepsilon} /(S W)$

$f_{\varphi}^{*}=f_{\varphi} /(S W)$

$F_{X}^{*}=F_{X} / W$

$F_{Y}^{*}=F_{Y} / W$

$h^{*}=h / c$

$K_{i j}^{*} \quad$ non-dimensional stiffness coefficients, $i, j=X, Y$

$p^{*}=p C^{2} /\left(\mu \omega R^{2}\right)$

$S=\mu \omega R L^{3} /\left(W C^{2}\right)$

$z^{*}=2 z / L$

Subscripts and superscripts

gz gradient on $z$ direction

s stability state

$\varepsilon \quad$ component on eccentric direction

$\varphi \quad$ component perpendicular to eccentric direction

* non-dimensional parameter

of rotor-bearing systems. Wang and Khonsari [13] proposed a method to calculate the dynamic coefficients to obtain an improved physical meaning. It is known that oil stiffness and damping characteristics are important for linear threshold speed and stability boundary of hydrodynamic bearings.

The coordinate system applied in the general Reynolds equation is polar coordinates. Thus, the oil film force is always obtained in polar coordinates and the stiffness and dampness parameters can be easily obtained in polar coordinates. While discussing linear threshold speed and stability boundary of hydrodynamic bearings, the stiffness and dampness parameters in rectangular coordinates are required. As per our understanding, the coordinate system applied in the previous study on linear threshold speed and stability boundary was either polar coordinates or rectangular coordinates for the complete calculation, which com- plicated the analysis. To address this complication, the transformation between polar and rectangular coordinates of journal bearing stiffness and dampness parameters is discussed in this study. The stiffness and dampness parameters are first calculated in polar coordinates, and then, converted into rectangular coordinates. Further, the stiffness and dampness parameters in rectangular coordinates can be applied to calculate linear threshold speed and stability boundary of hydrodynamic bearings.

\section{Transformation between polar and rectangular coordinates}

Figure 1 shows the journal bearing structure. The journal rotates in an anticlockwise direction with an angular speed $\omega$ and a radius $R$. The radial clearance is expressed as $C$, eccentricity is expressed as $e$, angular 


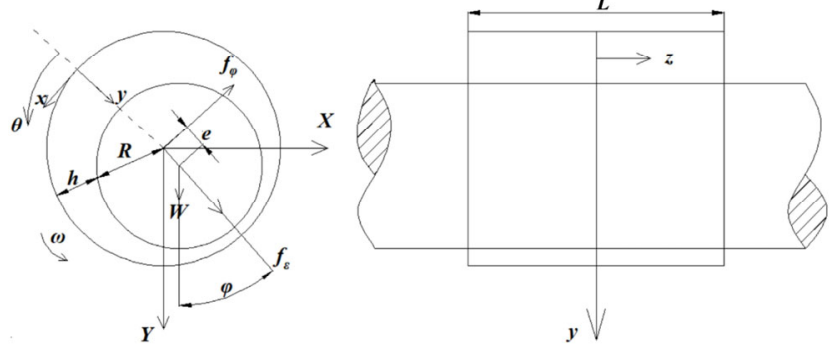

Fig. 1 Schematic diagram of the journal bearing.

coordinate of the bearing surface is expressed as $\theta$, and film thickness is expressed as $h=C+e \cos \theta$. Further, $\varphi$ is the attitude angle, $f_{\varphi}$ is the fluid force component in the direction of attitude angle, and $f_{\varepsilon}$ is a component in the eccentric direction. The external load on the journal is $W$.

The non-dimensional parameters are as follows:

$$
\begin{aligned}
x & =R \theta, z=\frac{L}{2} z^{*}, \alpha=k\left(\frac{\mu R \omega}{C}\right)^{2}, h=C h^{*}=C(1+\varepsilon \cos \theta), \\
e & =C \varepsilon, p=\mu \omega\left(\frac{R}{C}\right)^{2} p^{*}, \beta=\frac{D}{L}, \\
\dot{\varepsilon} & =\frac{1}{\omega} \frac{\mathrm{d} \varepsilon}{\mathrm{d} t}, \dot{\varphi}=\frac{1}{\omega} \frac{\mathrm{d} \varphi}{\mathrm{d} t}, S=\frac{\mu \omega R^{3} L}{W C^{2}}, f_{\varepsilon}=\frac{\mu \omega R^{3} L}{C^{2}} f_{\varepsilon}^{*}, \\
f_{\varphi} & =\frac{\mu \omega R^{3} L}{C^{2}} f_{\varphi}^{*}, F_{X}=F_{X}^{*} W, F_{Y}=F_{Y}^{*} W
\end{aligned}
$$

Threshold speed is an important parameter for the stability of the oil film bearing, and the non-dimensional linear threshold speed of the journal bearing $\omega_{s}^{*}$ is as follows [5, 8].

$$
\sqrt{\frac{\omega_{s}^{*}=}{\frac{K_{X X}^{*} C_{Y Y}^{*}+K_{Y Y}^{*} C_{X X}^{*}-K_{X Y}^{*} C_{Y X}^{*}-K_{Y X}^{*} C_{X Y}^{*}}{C_{X X}^{*}+C_{Y Y}^{*}}-\left(K_{X X}^{*}+K_{Y Y}^{*}\right)+}}
$$

where $K_{i j}^{*}$ and $C_{i j}^{*}(i, j=X, Y)$ are the non-dimensional stiffness and dampness parameters of the bearing in rectangular coordinates, respectively.

Although the results of oil film forces in journal bearings have always been expressed in polar coordinates, in this study, the transformation between polar and rectangular coordinates of journal bearing stiffness and dampness parameters is discussed as follows:

The non-dimensional oil film forces are expended in the $X$ and $Y$ directions as a first-order Taylor series approximation, as shown in Eq. (3).

$$
\left[\begin{array}{l}
\mathrm{d} F_{X}^{*} \\
\mathrm{~d} F_{Y}^{*}
\end{array}\right]=-\boldsymbol{K}_{\mathrm{r}}\left[\begin{array}{l}
\mathrm{d} X^{*} \\
\mathrm{~d} Y^{*}
\end{array}\right]-\mathbf{C}_{\mathrm{r}}\left[\begin{array}{c}
\mathrm{d} \dot{X}^{*} \\
\mathrm{~d} \dot{Y}^{*}
\end{array}\right]
$$

where $K_{\mathrm{r}}$ and $C_{\mathrm{r}}$ are the non-dimensional stiffness and dampness matrixes in rectangular coordinates, respectively. The expressions for $\boldsymbol{K}_{\mathrm{r}}$ and $\boldsymbol{C}_{\mathrm{r}}$ are as follows:

$$
\begin{gathered}
\boldsymbol{K}_{\mathrm{r}}=\left[\begin{array}{ll}
K_{X X}^{*} & K_{X Y}^{*} \\
K_{Y X}^{*} & K_{Y Y}^{*}
\end{array}\right]=\left[\begin{array}{ll}
\frac{\partial F_{X}^{*}}{\partial X^{*}} & \frac{\partial F_{X}^{*}}{\partial Y^{*}} \\
\frac{\partial F_{Y}^{*}}{\partial X^{*}} & \frac{\partial F_{Y}^{*}}{\partial Y^{*}}
\end{array}\right], \\
\boldsymbol{C}_{\mathrm{r}}=\left[\begin{array}{ll}
C_{X X}^{*} & C_{X Y}^{*} \\
C_{Y X}^{*} & C_{Y Y}^{*}
\end{array}\right]=\left[\begin{array}{ll}
\frac{\partial F_{X}^{*}}{\partial \dot{X}^{*}} & \frac{\partial F_{X}^{*}}{\partial \dot{Y}^{*}} \\
\frac{\partial F_{Y}^{*}}{\partial \dot{X}^{*}} & \frac{\partial F_{Y}^{*}}{\partial \dot{Y}^{*}}
\end{array}\right]
\end{gathered}
$$

In Fig. 1, the non-dimensional resultant forces in the $X$ and $Y$ directions are as follows:

$$
\begin{gathered}
F_{X}^{*}\left(X^{*}, Y^{*}, \dot{X}^{*}, \dot{Y}^{*}\right)=S\left(f_{\varepsilon}^{*} \sin \varphi+f_{\varphi}^{*} \cos \varphi\right) \\
F_{Y}^{*}\left(X^{*}, Y^{*}, \dot{X}^{*}, \dot{Y}^{*}\right)=S\left(f_{\varepsilon}^{*} \cos \varphi-f_{\varphi}^{*} \sin \varphi\right)+1
\end{gathered}
$$

where $S$ is the Sommerfeld number of the bearing. At the equilibration position, the expression of $S$ is

$$
S=\left(\frac{1}{\sqrt{f_{\varepsilon}^{* 2}+f_{\varphi}^{* 2}}}\right)_{s}
$$

where the subscript $s$ denotes the equilibrium position.

The differential of Eqs. (5) and (6) is calculated as

$$
\left[\begin{array}{l}
\mathrm{d} F_{X}^{*} \\
\mathrm{~d} F_{Y}^{*}
\end{array}\right]=S \boldsymbol{A}\left(\left[\begin{array}{l}
\mathrm{d} f_{\varepsilon}^{*} \\
\mathrm{~d} f_{\varphi}^{*}
\end{array}\right]-\left[\begin{array}{l}
f_{\varphi}^{*} \mathrm{~d} \varphi \\
-f_{\varepsilon}^{*} \mathrm{~d} \varphi
\end{array}\right]\right)
$$

where $A$ is a transformation matrix as follows:

$$
\boldsymbol{A}=\left[\begin{array}{ll}
\sin \varphi & \cos \varphi \\
\cos \varphi & -\sin \varphi
\end{array}\right]
$$

The differential of non-dimensional oil film forces 
in the $\varepsilon$ and $\varphi$ directions are

$$
\left[\begin{array}{l}
\mathrm{d} f_{\varepsilon}^{*} \\
\mathrm{~d} f_{\varphi}^{*}
\end{array}\right]=\boldsymbol{K}_{p}\left[\begin{array}{l}
\mathrm{d} \varepsilon \\
\varepsilon \mathrm{d} \varphi
\end{array}\right]+\boldsymbol{C}_{p}\left[\begin{array}{l}
\mathrm{d} \dot{\varepsilon} \\
\varepsilon \mathrm{d} \dot{\varphi}
\end{array}\right]
$$

where $\boldsymbol{K}_{p}$ and $\boldsymbol{C}_{p}$ are the non-dimensional stiffness and dampness matrixes in rectangular coordinates, respectively. The expressions of $\boldsymbol{K}_{p}$ and $\mathbf{C}_{p}$ are

$$
\boldsymbol{K}_{p}=\left[\begin{array}{ll}
\frac{\partial f_{\varepsilon}^{*}}{\partial \varepsilon} & \frac{\partial f_{\varepsilon}^{*}}{\varepsilon \partial \varphi} \\
\frac{\partial f_{\varphi}^{*}}{\partial \varepsilon} & \frac{\partial f_{\varphi}^{*}}{\varepsilon \partial \varphi}
\end{array}\right], \quad \boldsymbol{C}_{p}=\left[\begin{array}{ll}
\frac{\partial f_{\varepsilon}^{*}}{\partial \dot{\varepsilon}} & \frac{\partial f_{\varepsilon}^{*}}{\varepsilon \partial \dot{\varphi}} \\
\frac{\partial f_{\varphi}^{*}}{\partial \dot{\varepsilon}} & \frac{\partial f_{\varphi}^{*}}{\varepsilon \partial \dot{\varphi}}
\end{array}\right]
$$

By substituting Eq. (10) in Eq. (8), we get

$$
\left[\begin{array}{l}
\mathrm{d} F_{X}^{*} \\
\mathrm{~d} F_{Y}^{*}
\end{array}\right]=S\left[\begin{array}{ll}
\sin \varphi & \cos \varphi \\
\cos \varphi & -\sin \varphi
\end{array}\right]\left\{\left(\boldsymbol{K}_{p}+\boldsymbol{K}_{p 0}\right)\left[\begin{array}{l}
d \varepsilon \\
\varepsilon d \varphi
\end{array}\right]+\boldsymbol{C}_{p}\left[\begin{array}{l}
\mathrm{d} \dot{\varepsilon} \\
\varepsilon \mathrm{d} \dot{\varphi}
\end{array}\right]\right\}
$$

where $\boldsymbol{K}_{p 0}$ is the non-dimensional stiffness correction matrix as follows:

$$
\boldsymbol{K}_{p 0}=\left[\begin{array}{rr}
0 & -\frac{f_{\varphi}^{*}}{\varepsilon} \\
0 & \frac{f_{\varepsilon}^{*}}{\varepsilon}
\end{array}\right]
$$

The coordinate transformations between $\left(X^{*}, Y^{*}\right)$ and $(\varepsilon, \varphi)$ at the equilibrium position are

$$
\begin{aligned}
& {\left[\begin{array}{l}
\mathrm{d} X^{*} \\
\mathrm{~d} Y^{*}
\end{array}\right]=\boldsymbol{A}\left[\begin{array}{l}
\mathrm{d} \varepsilon \\
\varepsilon \mathrm{d} \varphi
\end{array}\right]} \\
& {\left[\begin{array}{l}
\mathrm{d} \dot{X}^{*} \\
\mathrm{~d} \dot{Y}^{*}
\end{array}\right]=\boldsymbol{A}\left[\begin{array}{l}
\mathrm{d} \dot{\varepsilon} \\
\varepsilon \mathrm{d} \dot{\varphi}
\end{array}\right]}
\end{aligned}
$$

When Eqs. (14) and (15) are substituted in Eq. (12), the expression obtained is

$$
\left[\begin{array}{l}
\mathrm{d} F_{X}^{*} \\
\mathrm{~d} F_{Y}^{*}
\end{array}\right]_{s}=S \boldsymbol{A}\left\{\left(\boldsymbol{K}_{p}+\boldsymbol{K}_{p 0}\right) \boldsymbol{A}\left[\begin{array}{l}
\mathrm{d} X^{*} \\
\mathrm{~d} Y^{*}
\end{array}\right]+\boldsymbol{C}_{p} \boldsymbol{A}\left[\begin{array}{c}
\mathrm{d} \dot{X}^{*} \\
\mathrm{~d} \dot{Y}^{*}
\end{array}\right]\right\}
$$

From Eqs. (3) and (16), the stiffness and dampness coefficients of the oil film on Cartesian coordinate system at the equilibrium position are derived as follows:

$$
\boldsymbol{K}_{\mathrm{r}}=-S \boldsymbol{A}\left(\boldsymbol{K}_{p}+\boldsymbol{K}_{p 0}\right) \boldsymbol{A}
$$

$$
\mathbf{C}_{r}=-S A C_{p} A
$$

Equations (17) and (18) are the general transformation of the hydrodynamic journal bearing stiffness and dampness coefficients between polar and rectangular coordinates, respectively. The transformation validity for short journal bearings is discussed in Section 3.

\section{Verification and discussion}

When the short-bearing approximate is applied with half-Sommerfeld boundary conditions, the nondimensional oil film forces of the bearing are as follows $[6,13]$.

$$
\begin{gathered}
f_{\varepsilon}^{*}=-\left(\frac{\varepsilon^{2}(1-2 \dot{\varphi})}{\left(1-\varepsilon^{2}\right)^{2}}+\frac{\pi\left(1+2 \varepsilon^{2}\right) \dot{\varepsilon}}{2\left(1-\varepsilon^{2}\right)^{\frac{5}{2}}}\right) \\
f_{\varphi}^{*}=\frac{\pi \varepsilon(1-2 \dot{\varphi})}{4\left(1-\varepsilon^{2}\right)^{\frac{3}{2}}}+\frac{2 \varepsilon \dot{\varepsilon}}{\left(1-\varepsilon^{2}\right)^{2}}
\end{gathered}
$$

At equilibrium position, the stiffness and dampness parameters of the bearing in polar coordinates are

$$
\begin{gathered}
K_{\varepsilon \varepsilon}^{*}=\frac{\partial f_{\varepsilon}^{*}}{\partial \varepsilon}=-\frac{2 \varepsilon\left(1+\varepsilon^{2}\right)}{\left(1-\varepsilon^{2}\right)^{3}}, K_{\varepsilon \varphi}^{*}=\frac{\partial f_{\varepsilon}^{*}}{\varepsilon \partial \varphi}-\frac{f_{\varphi}^{*}}{\varepsilon}=-\frac{\pi}{4\left(1-\varepsilon^{2}\right)^{\frac{3}{2}}}, \\
K_{\varphi \varepsilon}^{*}=\frac{\partial f_{\varphi}^{*}}{\partial \varepsilon}=\frac{\pi\left(1+2 \varepsilon^{2}\right)}{4\left(1-\varepsilon^{2}\right)^{\frac{5}{2}}}, K_{\varphi \varphi}^{*}=\frac{\partial f_{\varphi}^{*}}{\varepsilon \partial \varphi}+\frac{f_{\varepsilon}^{*}}{\varepsilon}=-\frac{\varepsilon}{\left(1-\varepsilon^{2}\right)^{2}}
\end{gathered}
$$

$$
\begin{aligned}
& D_{\varepsilon \varepsilon}^{*}=\frac{\partial f_{\varepsilon}^{*}}{\partial \dot{\varepsilon}}=-\frac{\pi\left(1+2 \varepsilon^{2}\right)}{2\left(1-\varepsilon^{2}\right)^{\frac{5}{2}}}, D_{\varepsilon \varphi}^{*}=\frac{\partial f_{\varepsilon}^{*}}{\varepsilon \partial \dot{\varphi}}=\frac{2 \varepsilon}{\left(1-\varepsilon^{2}\right)^{2}}, \\
& D_{\varphi \varepsilon}^{*}=\frac{\partial f_{\varphi}^{*}}{\partial \dot{\varepsilon}}=\frac{2 \varepsilon}{\left(1-\varepsilon^{2}\right)^{2}}, \quad D_{\varphi \varphi}^{*}=\frac{\partial f_{\varphi}^{*}}{\varepsilon \partial \dot{\varphi}}=-\frac{\pi}{2\left(1-\varepsilon^{2}\right)^{\frac{3}{2}}}
\end{aligned}
$$

Further, the attitude angle at the equilibrium position is

$$
\varphi=\tan ^{-1}\left(\frac{f_{\varphi}^{*}}{f_{\varepsilon}^{*}}\right)=\tan ^{-1}\left[\frac{\pi\left(1-\varepsilon^{2}\right)}{4 \varepsilon}\right]
$$


When Eqs. (7) and (21)-(23) are substituted in Eqs. (17)-(18), the stiffness and dampness parameters in rectangular coordinates are

$$
\begin{aligned}
K_{X X}^{*}= & -S\left(\sin ^{2} \varphi K_{\varepsilon \varepsilon}^{*}+\sin \varphi \cos \varphi\left(K_{\varphi \varepsilon}^{*}+K_{\varepsilon \varphi}^{*}\right)+\cos ^{2} \varphi K_{\varphi \varphi}^{*}\right)= \\
& \frac{4\left[2 \pi^{2}+\left(16-\pi^{2}\right) \varepsilon^{2}\right]}{\left[\pi^{2}+\left(16-\pi^{2}\right) \varepsilon^{2}\right]^{\frac{3}{2}}} \\
K_{X Y}^{*}= & -S\left(\cos ^{2} \varphi K_{\varphi \varepsilon}^{*}+\sin \varphi \cos \varphi\left(K_{\varepsilon \varepsilon}^{*}-K_{\varphi \varphi}^{*}\right)-\sin ^{2} \varphi K_{\varepsilon \varphi}^{*}\right)= \\
& \frac{-\pi\left[-\pi^{2}+2 \pi^{2} \varepsilon^{2}+\left(16-\pi^{2}\right) \varepsilon^{4}\right]}{\varepsilon\left(1-\varepsilon^{2}\right)^{\frac{1}{2}}\left[\pi^{2}+\left(16-\pi^{2}\right) \varepsilon^{2}\right]^{\frac{3}{2}}} \\
K_{Y X}^{*}= & -S\left(\cos ^{2} \varphi K_{\varepsilon \varphi}^{*}+\sin \varphi \cos \varphi\left(K_{\varepsilon \varepsilon}^{*}-K_{\varphi \varphi}^{*}\right)-\sin ^{2} \varphi K_{\varphi \varepsilon}^{*}\right)= \\
& \frac{-\pi\left[\pi^{2}+\left(32+\pi^{2}\right) \varepsilon^{2}+2\left(16-\pi^{2}\right) \varepsilon^{4}\right]}{\varepsilon\left(1-\varepsilon^{2}\right)^{\frac{1}{2}}\left[\pi^{2}+\left(16-\pi^{2}\right) \varepsilon^{2}\right]^{\frac{3}{2}}} \\
K_{Y Y}^{*}= & -S\left(\cos ^{2} \varphi K_{\varepsilon \varepsilon}^{*}-\sin \varphi \cos \varphi\left(K_{\varphi \varepsilon}^{*}+K_{\varepsilon \varphi}^{*}\right)+\sin ^{2} \varphi K_{\varphi \varphi}^{*}\right)= \\
& \frac{4\left[\pi^{2}+\left(32+\pi^{2}\right) \varepsilon^{2}+2\left(16-\pi^{2}\right) \varepsilon^{4}\right]}{\left(1-\varepsilon^{2}\right)\left[\pi^{2}+\left(16-\pi^{2}\right) \varepsilon^{2}\right]^{\frac{3}{2}}}
\end{aligned}
$$

$D_{X X}^{*}=-S\left(\sin ^{2} \varphi D_{\varepsilon \varepsilon}^{*}+\sin \varphi \cos \varphi\left(D_{\varphi \varepsilon}^{*}+D_{\varepsilon \varphi}^{*}\right)+\cos ^{2} \varphi D_{\varphi \varphi}^{*}\right)=$

$$
\frac{2 \pi\left(1-\varepsilon^{2}\right)^{\frac{1}{2}}\left[\pi^{2}+2\left(\pi^{2}-8\right) \varepsilon^{2}\right]}{\varepsilon\left[\pi^{2}+\left(16-\pi^{2}\right) \varepsilon^{2}\right]^{\frac{3}{2}}}
$$

$D_{X Y}^{*}=-S\left(\cos ^{2} \varphi D_{\varphi \varepsilon}^{*}+\sin \varphi \cos \varphi\left(D_{\varepsilon \varepsilon}^{*}-D_{\varphi \varphi}^{*}\right)-\sin ^{2} \varphi D_{\varepsilon \varphi}^{*}\right)=$

$$
-\frac{8\left[\pi^{2}+2\left(\pi^{2}-8\right) \varepsilon^{2}\right]}{\left[\pi^{2}+\left(16-\pi^{2}\right) \varepsilon^{2}\right]^{\frac{3}{2}}}
$$

$D_{Y X}^{*}=-S\left(\cos ^{2} \varphi D_{\varepsilon \varphi}^{*}+\sin \varphi \cos \varphi\left(D_{\varepsilon \varepsilon}^{*}-D_{\varphi \varphi}^{*}\right)-\sin ^{2} \varphi D_{\varphi \varepsilon}^{*}\right)=$

$$
-\frac{8\left[\pi^{2}+2\left(\pi^{2}-8\right) \varepsilon^{2}\right]}{\left[\pi^{2}+\left(16-\pi^{2}\right) \varepsilon^{2}\right]^{\frac{3}{2}}}
$$

$D_{Y Y}^{*}=-S\left(\cos ^{2} \varphi D_{\varepsilon \varepsilon}^{*}-\sin \varphi \cos \varphi\left(D_{\varphi \varepsilon}^{*}+D_{\varepsilon \varphi}^{*}\right)+\sin ^{2} \varphi D_{\varphi \varphi}^{*}\right)=$

$$
\frac{2 \pi\left[\pi^{2}+2\left(24-\pi^{2}\right) \varepsilon^{2}+\pi^{2} \varepsilon^{4}\right]}{\varepsilon\left(1-\varepsilon^{2}\right)^{\frac{1}{2}}\left[\pi^{2}+\left(16-\pi^{2}\right) \varepsilon^{2}\right]^{\frac{3}{2}}}
$$

Further, the obtained results are in accordance with
Ref. [5]. Therefore, the general transformation of the hydrodynamic journal bearing stiffness and dampness coefficients between polar and rectangular coordinates, as expressed by Eqs. (17) and (18), are verified.

\section{Conclusions}

The derived expression denotes the general transformation between polar and rectangular coordinates of journal bearing stiffness and dampness parameters. The validity of this study is confirmed with the use of short journal bearings. Further, this transformation is also suitable for long journal and finite journal bearings.

\section{Acknowledgements}

This research is supported by science and technology research project of Hubei provincial department of education (No. Q20192603) and doctoral research fund of Hubei University of Arts and Science (No. 2059023).

Open Access: This article is licensed under a Creative Commons Attribution 4.0 International License, which permits use, sharing, adaptation, distribution and reproduction in any medium or format, as long as you give appropriate credit to the original author(s) and the source, provide a link to the Creative Commons licence, and indicate if changes were made.

The images or other third party material in this article are included in the article's Creative Commons licence, unless indicated otherwise in a credit line to the material. If material is not included in the article's Creative Commons licence and your intended use is not permitted by statutory regulation or exceeds the permitted use, you will need to obtain permission directly from the copyright holder.

To view a copy of this licence, visit http://creativecommons.org/licenses/by/4.0/.

\section{References}

[1] Holmes R. The vibration of a rigid shaft on short sleeve bearings. J Mech Eng Sci 2(4): 337-341 (1960)

[2] Hahn E J. The excitability of flexible rotors in short sleeve bearings. J Lubr Technol 97(1): 105-115 (1975) 
[3] Hashimoto H, Wada S. Dynamic behavior of unbalanced rigid shaft supported on turbulent journal bearings - theory and experiment. J Tribol 112(2): 404-408 (1990)

[4] Khonsari M M. On the self-excited whirl orbits of a journal in a sleeve bearing lubricated with micropolar fluids. Acta Mech 81(3-4): 235-244 (1990)

[5] Khonsari M M, Chang Y J. Stability boundary of non-linear orbits within clearance circle of journal bearings. J Vib Acoust 115(3): 303-307 (1993)

[6] Huang Y, Tian Z X, Chen R C, Cao H Y. A simpler method to calculate instability threshold speed of hydrodynamic journal bearings. Mech Mach Theory 108: 209-216 (2017)

[7] Lin J R. Linear stability analysis of rotor-bearing system: Couple stress fluid model. Comput Struct 79(8): 801-809 (2001)

[8] Lin J R, Li P J, Hung T C, Liang L J. Nonlinear stability boundary of journal bearing systems operating with nonNewtonian couple stress fluids. Tribol Int 71: 114-119 (2014)

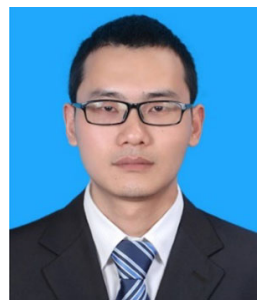

Zhuxin TIAN. He received his bachelor degree in mechanical design manufacturing and automation in 2009 from Huazhong University of Science and Technology, Wuhan, China. He has obtained his Ph.D.

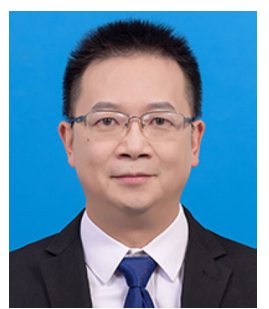

Yu HUANG. He received his bachelor degree in machinery manufacturing process and equipment in 1993 from Huazhong University of Science and Technology, Wuhan, China. He received his M.S. and Ph.D. degrees in automotive engineering and mechanical design manufacturing and automation from Huazhong University of Science and Technology in 2000 and 2004, respectively. After then, he joined
[9] Kushare P B, Sharma S C. Nonlinear transient stability study of two lobe symmetric hole entry worn hybrid journal bearing operating with non-Newtonian lubricant. Tribol Int 69: 84-101 (2014)

[10] Lund J W, Thomsen K K. A calculation method and data for the dynamic coefficients of oil-lubricated journal bearings. In Topics in Fluid Film Bearing and Rotor Bearing System Design and Optimization. New York, 1978: 1-28.

[11] Jang G H, Kim Y J. Calculation of dynamic coefficients in a hydrodynamic bearing considering five degrees of freedom for a general rotor-bearing system. $J$ Tribol 121(3): 499-505 (1999)

[12] Crooijmans M T M, Brouwers H J H, Van Campen D H, De Kraker A. Limit cycle predictions of a nonlinear journalbearing system. J Eng Ind 112(2): 168-171 (1990)

[13] Wang J K, Khonsari M M. A new derivation for journal bearing stiffness and damping coefficients in polar coordinates. J Sound Vib 290(1-2): 500-507 (2006)

degree in mechatronic engineering from Huazhong University of Science and Technology in 2018. After then, he joined Hubei University of Arts and Science as a lecturer. His research interests include hydrostatic and hydrodynamic bearings.

Huazhong University of Science and Technology as a lecturer. His current position is a professor and the deputy director of the National engineering research center for digitalization of manufacturing equipment. And he joined Hubei University of Arts and Science as an adjunct professor in 2019. His research areas cover high performance hydrostatic bearing, laser fine processing technology and equipment and highpower laser cutting and welding equipment. 